\title{
“ESPERANDO JUSTICIA". TRAUMA PSÍQUICO, TEMPORALIDAD Y MOVILIZACIÓN POLÍTICA EN LA ARGENTINA ACTUAL
}

\author{
"Waiting for justice". Trauma, temporality and political mobilization in \\ contemporary Argentina
}

\author{
Diego Zenobi* \\ * Universidad de Buenos Aires (Argentina) \\ diego.zenobi@gmail.com
}

Palabras clave Trauma psíquico Temporalidad Víctimas Movilización política

\begin{abstract}
Resumen
En el mundo contemporáneo la condición de víctima ha cobrado centralidad como vector de identificación colectiva, y el trauma psíquico se ha constituido en un lenguaje razonable para comunicar el sufrimiento. Las víctimas de conflictos bélicos, violencia de género, catástrofes, etc., ponen en juego categorías como trauma o estrés postraumático para exigir derechos. Este es el caso de las víctimas de un incendio ocurrido en Buenos Aires, que durante años se movilizaron exigiendo que se realice el juicio penal para castigar a los responsables del hecho. Como sujetos de la política pública de atención en salud mental, se vincularon con los psicólogos y psiquiatras de un servicio estatal de estrés postraumático. A su vez, un grupo de profesionales psi con reconocidas trayectorias de compromiso con los derechos humanos apoyaron su causa. Apelando a la conexión entre trauma e impunidad, denunciaron al Estado por sumir a las víctimas en una larga y penosa espera por la realización del juicio. Al hacerlo, dejaron expuesta una relación entre el proceso psíquico y el proceso penal. Aquí retomo la "antropología del tiempo" para analizar esta coordinación que se dio durante la espera entre procesos de diferente naturaleza. Para ello, me apoyo en los materiales producidos durante un trabajo de campo etnográfico de tres años con las víctimas. Concluyo que los profesionales psi y el Estado tuvieron un rol central en la definición del hecho como traumático y en la certificación de la veracidad del sufrimiento de las víctimas que, al mismo tiempo, lo confrontaban.
\end{abstract}

\begin{abstract}
$\underline{\text { Abstract }}$
In the contemporary world victimhood has become a vector of collective identity, and psychic trauma has become a reasonable language for communicating their suffering. The victims of war, genderbased violence, disasters, etc., come into play psychological categories such as trauma or posttraumatic stress disorder to demand their rights. This is what happened with the victims of a fire occurred in Buenos Aires, who for several years mobilized asking for the punishment of those responsible of the incident. As related with public policy mental health care, victims were treated by psychologists and psychiatrists of a State service concerned with post-traumatic stress disorder. At the same time, a group of 'psi' professionals with recognized trajectories concerned with human rights supported their cause. Calling attention to the connection between trauma and impunity, they sued the State for the long and painful wait of the victims until the completion of the criminal trial. By doing so, they exposed a relationship between the psychological process and the criminal proceedings. Based on the materials produced during a three-year ethnographic fieldwork with the victims, I return here to the "anthropology of time" in order to analyze this coordination that occurred during the waiting time between those processes of different nature - the psychological and the criminal one-. I conclude that psi professionals and the State played a central role in defining the event as traumatic and in certifying the accuracy of victims' suffering that, at the same time, confronted it.
\end{abstract}

\section{Keywords}

Psychic trauma Temporality

Victims

Political mobilization

Zeboni, D. (2017). "Esperando justicia". Trauma psíquico, temporalidad y movilización política en la argentina actual. Papeles del CEIC. International Journal on Collective Identity Research, vol. 2017/1, papel 170, CEIC (Centro de Estudios sobre la Identidad Colectiva), UPV/EHU Press, http://dx.doi.org/10.1387/pceic.16921 


\section{INTRODUCCIÓN}

La reflexión que presento en este artículo se inició con un curioso episodio acaecido durante mi trabajo de campo. El mismo se desarrolló en un colectivo conformado por las víctimas de un incendio ocurrido en Buenos Aires en 2004 durante un recital de rock, en el que murieron intoxicados 194 jóvenes y más de 3.000 sobrevivieron con diferentes tipos de daños. Una tarde de 2008 el movimiento de víctimas organizó una conferencia de prensa para denunciar que las autoridades judiciales estaban retrasando el inicio del juicio penal para condenar a los responsables de su sufrimiento. Al llegar a la conferencia de prensa me sorprendí al encontrar que no eran los familiares de los muertos, quienes llevaban la voz pública de la lucha, ni los sobrevivientes del incendio quienes hablarían a los medios de comunicación. En cambio, se trataba de un grupo de especialistas de la salud mental: psicólogos, psiquiatras y psicoanalistas, eran los protagonistas del evento. Ellos denunciaron que ya no podía continuar la espera a la que estaban sometidas las víctimas traumatizadas: ellas necesitaban reparación y ello sería posible sólo cuando se realizara el juicio penal y se condenara a los responsables del hecho. En ese marco, categorías como trauma, duelo patológico y estrés postraumático se revelaron como eficaces herramientas para la movilización política y la impugnación al Estado.

En este artículo recupero los materiales producidos durante mi trabajo de campo etnográfico entre los sobrevivientes y familiares de los fallecidos en el incendio. Se trata de mis notas de campo elaboradas a partir de la observación participante prolongada en el terreno, de materiales periodísticos, así como de documentos y producciones elaboradas por el movimiento de víctimas y agencias estatales.

\section{UN INCENDIO Y 194 MUERTES TRAUMÁTICAS}

En el contexto de consolidación de las exigencias de ciudadanía, hace varias décadas que el mundo contemporáneo está asistiendo a una ampliación del lugar social ocupado por la figura de la víctima. Por un lado, los hechos que generan víctimas son cada vez más diversos: puede tratarse de catástrofes naturales, guerras, desastres tecnológicos, terrorismo de 
Estado, pero también de situaciones de abuso sexual, violencia de género, mobbing, bullying, etc. Por otra parte, la noción de víctima se ha expandido en otro sentido ya que cada vez con mayor frecuencia, amigos, parejas y familiares de los afectados directos reclaman ser reconocidos de ese modo. Esa ampliación de la noción de víctima ha contribuido a la conformación de comunidades de personas que reivindican reconocimiento y reparación a través de las narraciones de sufrimiento que ponen en juego en el espacio público. La centralidad de la condición de víctima en las sociedades contemporáneas debe ser pensada en relación a su capacidad para legitimar demandas y exigencias de los más diversos sectores de la ciudadanía (Sarti, 2011).

El incendio del estadio conocido como República Cromañón fue producto del impacto de un fuego de artificio en el revestimiento acústico del techo. Con las ventilaciones de aire y las puertas de emergencia clausuradas ilegalmente, el humo tóxico se esparció rápidamente por el lugar sin dar la posibilidad de escapar a los miles de asistentes. Los decesos causados por la asfixia y el aplastamiento, muertes violentas e inesperadas, contrastan con otras formas de morir que son asumidas como previsibles y naturales (Elias, 1980). Por ello, fueron entendidas como muertes traumáticas, del mismo modo que las consecuencias que tuvieron para los sobrevivientes y los familiares de los fallecidos. Estos últimos debieron enfrentarse con el carácter prematuro del fallecimiento de los hijos que alteró el ciclo natural de la vida ya que los jóvenes murieron antes que los viejos. Apenas días después del hecho las víctimas comenzaron a organizar multitudinarias movilizaciones por las calles de Buenos Aires de las que participaban sobrevivientes, amigos y activistas de diversos partidos políticos, exigiendo que se condenara a los responsables penales y políticos del incendio. Como parte de sus iniciativas de lucha ese movimiento de demanda de justicia en el que los padres de los muertos tenían la voz pública legítima, el movimiento Cromañón construyó un memorial conocido como "el santuario" que se transformaría en el punto de encuentro y centro simbólico de la protesta.

En el discurso público acerca del sufrimiento de las víctimas hubo un uso extendido de categorías psicológicas como trauma, duelo patológico o estrés postraumático. La circulación de categorias psi en este tipo de situaciones no es una particularidad del caso que aquí me ocupa. En palabras de Fassin y Rechtman (2007) estaríamos ante un "imperio del trauma". Esto es, ante la difusión del lenguaje del trauma psíquico como un 
modo poderoso y novedoso de entender el sufrimiento de las poblaciones, que transformó la noción contemporánea de víctima tal como la entendíamos hasta la primera mitad del siglo XX.

En el presente artículo indago en las relaciones establecidas entre especialistas en salud mental y las víctimas del incendio de Cromañón que movilizaron las categorías psi en el espacio público argentino para ser socialmente reconocidas pero, sobre todo, para demandar por sus derechos. A través de las relaciones de interdependencia que tramaron unos y otros, dieron vida a una configuración social particular (Elias, 1982) a través de la cual se modeló el carácter traumático del evento. Considero que las formas de acción y representación de las víctimas y de los expertos con los que se relacionaron, no deben ser entendidas simplemente como respuestas a determinados condicionantes y factores externos que las han golpeado generando dolor y sufrimiento, sino como vehículos de constitución del evento como traumático (Visacovsky, 2009). Tal carácter es producto de un proceso colectivo de definición sometido a ciertas condiciones locales en el marco de las cuales esa etiqueta fue socialmente producida.

A lo largo de este trabajo, analizo en primer lugar el papel de un servicio de estrés postraumático de un hospital, dispositivo estatal central destinado a la atención clínica a las víctimas del incendio. En segundo lugar, abordo la denuncia de la espera por la realización del juicio penal llevada adelante por profesionales psi comprometidos con la lucha del movimiento Cromañón. Como se ha destacado desde la "antropología del tiempo" (Bear, 2014; Gell, 1992), en ciertas circunstancias sociales la temporalidad se vuelve una cuestión de especial relevancia para las personas. Sostendré contra Auyero (2012) que, en este caso, la im-paciencia de Estado desembocó en crítica e impugnación al poder. Sobre esa base, mostraré luego cómo las propias víctimas pusieron en juego categorías psi dejando expuesta una relación entre el proceso psíquico y el proceso penal durante el tiempo de espera. Finalmente, concluyo que el Estado y sus profesionales de la salud mental tuvieron un rol central en la definición de lo sucedido como un hecho traumático y certificaron la veracidad del sufrimiento de las víctimas que, al mismo tiempo, lo denunciaban apelando a la conexión entre trauma e impunidad. 
3. EL ÉXITO DE LOS SABERES PSI: DIFUSIÓN GLOBAL Y CONDICIONES LOCALES

El Diagnostic and Statistical Manual of Mental Disorders (DSM), editado por la American Psychiatric Association (APA), es la guía de enfermedades y trastornos mentales más influyente a nivel global en el campo de la salud mental. Su edición del año 1980, el DSM III, implicó un cambio en el paradigma del conocimiento psi vigente tanto en los Estados Unidos como en el contexto internacional. Alli se ve cómo fue perdiendo centralidad la categoría de "trauma psíquico" en detrimento de la incorporación a la nosología del "Trastorno por Estrés Postraumático" (TEPT) como un desorden mental. El manual señala que el desorden se produce cuando las personas experimentan "un acontecimiento estresante y extremadamente traumático" (APA, 1994: 435) fuera del rango de la experiencia humana previsible y normal. Se citan ejemplos tales como los combates en el frente de guerra, agresiones sexuales, secuestros, torturas, catástrofes y desastres, accidentes automovilísticos, etc.

El largo proceso a través del cual la noción de "Trauma Psíquico", tema central del psicoanálisis fue dejando espacio en el DSM a la de "Estrés postraumático", estuvo relacionado con la acción de ciertos grupos de presión como los veteranos de la guerra de Vietnam y el movimiento feminista, así como con las luchas en el campo psi norteamericano durante los años $1970^{1}$. A diferencia de la anterior, esta categoría incluye el dialogo con la neurobiología, la terapéutica farmacológica y los tratamientos cognitivo-conductuales. Según Russo y Venâncio (2006) en la versión previa del DSM, la hegemonía del psicoanálisis implicaba la afirmación de la dimensión moral de los sujetos sociales sobre la dimensión física. Hasta la década de los años 1980, en el manual, la noción de "neurosis traumática" ligaba, relacionaba, el evento traumático con la personalidad preexistente del afectado. Debía buscarse en ese antecedente si existía un terreno fértil para el desarrollo de un trauma psíquico que necesariamente

\footnotetext{
${ }^{1}$ De acuerdo con Young (1995), la inclusión del TEPT al DSM III estuvo relacionada con la convergencia de diferentes intereses y situaciones. Por un lado, deben considerarse las aspiraciones de ciertos grupos de presión, entre ellos las feministas y los veteranos de la guerra de Vietnam que durante la década de 1970 exigían reparación a causa de los problemas psíquicos generados por el enfrentamiento bélico. Por otro lado, ha de considerarse que durante esa década la psiquiatría estadounidense estaba emprendiendo una importante reorganización interna, política, teórica e institucional que incluyó la revisión de la nosología vigente con el objetivo de crear un nuevo marco conceptual para fortalecer el carácter científico del enfoque psiquiátrico (d'Halluin et al., 2004).
} 
estaba vinculado con esa biografía. Ese paradigma se rompe cuando en el DSM III la categoría de TEPT desplaza a aquella y pasa a enfatizar la importancia del evento externo como el principal agente causal del trauma psíquico, independientemente de la singularidad del paciente tan central para el psicoanálisis (d'Halluin et al., 2004; Latté y Rechtman, 2006).

EI TEPT es parte central de las "tecnologías del trauma" (Caple James, 2010) que ponen en juego los agentes que trabajan en organismos internacionales y ONGs que despliegan intervenciones humanitarias en todo el planeta. A través de esa categoría se instrumenta la atención mental a víctimas con el objetivo de diagnosticar y autenticar su padecimiento a través de prácticas médicas y rutinas burocráticas (Seagal, 2014). A partir de su inclusión en el DSM III y de la mano de aquellas intervenciones que se dan en contextos sociales extremadamente diferentes y en tragedias de origen totalmente diverso, el TEPT ha alcanzado difusión global.

Según el DSM IV, editado en el año 1994, entre los síntomas que caracterizan al TEPT pueden señalarse la reexperimentación persistente del acontecimiento traumático (con la consecuente evitación de los estímulos asociados al mismo), hiperactividad fisiológica que puede causar trastornos de sueño, etc. A los fines de este trabajo me resulta de especial interés destacar, siguiendo a Young, que el TEPT "es una enfermedad del tiempo. El desorden distintivo de la patología es que permite que la memoria pasada sea revivida en el presente, bajo la forma de imágenes intrusivas y pensamientos así como en la compulsión del paciente por reactualizar eventos pasados" (1995: 7). Esto es, frente a determinadas circunstancias, es posible el retorno del padecimiento. Dice el manual que el TEPT puede desencadenarse "cuando el individuo se expone a estímulos desencadenantes que recuerdan o simbolizan un aspecto del acontecimiento traumático (p. ej., aniversarios del suceso)" (APA, 1994: 435). Esta caracterización se fundamenta sobre la noción de trauma psíquico que se constituyó a partir de los desarrollos freudianos sobre la neurosis de guerra, y que se encuentra constituida en torno a dos momentos: el momento disruptivo y su, siempre posible, reactualización.

Bien entrada la segunda mitad del siglo $X X$, se da una importante reconversión que contribuye a vincular el trauma psíquico con una mirada positiva sobre las víctimas. Si hasta entonces quien portaba un trauma podía ser visto y etiquetado negativamente como un enfermo, a partir de entonces esa categoría tomó contornos justificatorios: en aquel momento 
histórico, "el trauma, liberado de la sospecha que desacreditaba a los individuos (soldados, trabajadores, mujeres violadas o niños abusados) que tenían síntomas, toma una valencia moral positiva" (Fassin, 2014: 167). A partir de entonces, cuando se habla de trauma no se hace referencia tanto a una enfermedad mental sino más bien a una actitud vista como normal frente a un hecho anormal. Quien lo padece es visto como un sujeto sano que ha sido puesto a prueba por un suceso que lo excede. Ya no es tanto el discurso sobre el padecimiento corporal el que legitima públicamente el sufrimiento en situaciones críticas, sino la psiquis la que viene a autenticar la gravedad de lo sufrido.

En el caso de la Argentina, la difusión de conceptos psi para explicar el sufrimiento tiene una historia muy específica. En primer término debe considerarse la amplia recepción local del psicoanálisis en el campo médico-psiquiátrico y en el espacio hospitalario público (Visacovsky, 2002), así como la expansión hegemónica de esa corriente en las carreras universitarias de psicología. Para Visacovsky (2009) el psicoanálisis es el denominador común de un cierto universo "psi" en el cual actores e instituciones se legitiman reconociendo su origen en el pensamiento psicoanalítico o posicionándose frente al mismo. Sin embargo, y esto es lo central, el psicoanálisis no sólo existe bajo modalidades institucionalizadas sino también como modos de actuar y pensar de muchos argentinos. Según Plotkin (2001), el lenguaje psicoanalítico ha penetrado en la cultura argentina como modelo para interpretar la condición humana. Según su trabajo, esto podría verse en que desde la década de 1950 tal lenguaje permea el mundo literario, académico e intelectual, la difusión a través de la prensa, las revistas de actualidad orientadas al público femenino, o los programas de televisión. Su éxito radica en que se ha transformado en sentido común de ciertos sectores medios urbanos (Visacovsky, 2009). Acaso sea por ello que los argentinos utilizamos conceptos de ese campo para interpretar desde el sufrimiento cotidiano hasta otras circunstancias con profundidad histórica como el terrorismo de Estado y la violencia política (Robben, 2005). Al respecto señala Plotkin que "conceptos como trauma, represión, neurosis e inconsciente han pervivido desde finales de los años 1950s e inclusive fueron adoptados por el régimen militar de 19761983" (2001: 221) ${ }^{2}$. En ese escenario caracterizado por una amplia difusión del lenguaje psi, los especialistas de ese campo son socialmente reconocidos como voces autorizadas para interpretar situaciones

\footnotetext{
${ }^{2}$ Todas las traducciones en el texto son propias. 
nacionales críticas como aquel pasado dictatorial o bien otras más recientes como la crisis socioeconómica ocurrida en 2001 en la Argentina (Plotkin y Visacovsky, 2007). De manera tal que en ese país, en el marco de esos procesos históricos y sociales específicos, las explicaciones psi se han convertido en un marco razonable, inteligible, una cierta teodicea secular que permite comprender el sufrimiento.

\section{La INTERVENCIÓN CLÍNICA: EL SERVICIO DE ESTRÉS POSTRAUMÁTICO}

Desde una preocupación durkheimniana interesada por la producción de representaciones colectivas, me pregunto por el papel del Estado en el proceso social de definición de las consecuencias del incendio como una cuestión traumática. Las políticas públicas son, en ese sentido, un locus privilegiado desde el cual realizar tal indagación.

Desde las intervenciones estatales orientadas a brindar ayuda y atención hospitalaria a familiares y sobrevivientes, se consideró necesario atender "los efectos traumáticos de la situación vivida" el Estado de la Ciudad de Buenos Aires lanzó una política pública llamada Programa de atención integral a las víctimas del 30 de diciembre de 2004. Con el objetivo de gestionar la demanda de las víctimas, la Dirección de Salud Mental del gobierno de la ciudad local puso en marcha la línea telefónica Salud Mental Responde. Desde alli se derivaban las consultas a los servicios de psicopatología de los hospitales de la ciudad. Durante todo el 2005, el primer año posterior al incendio, se atendieron en los hospitales referentes de salud mental 932 pacientes los cuales eran fundamentalmente sobrevivientes (84\%), pero también sus familiares (11\%) y familiares de las víctimas fatales (3\%) (Andújar, 2006).

Como parte del programa se creó un subsidio económico mensual. El mismo se proponía brindar un monto de dinero mensual a quienes a causa del incendio se encontraban en situación de vulnerabilidad como consecuencia "del impacto provocado por algún evento traumático y que les impide continuar o retomar sus tareas habituales" ${ }^{\prime 4}$. Así fue que la atención mental de sobrevivientes y familiares de los fallecidos no sólo tenía un aspecto terapéutico, sino que para acceder al subsidio para las víctimas que formaba parte del programa de atención ellos debían contar

\footnotetext{
${ }^{3}$ Decreto $N^{\circ} 2.120 / 2006$, Gobierno de la Ciudad de Buenos Aires.

${ }^{4}$ Decreto $N^{\circ}$ 692/2005, Gobierno de la Ciudad de Buenos Aires. 
con certificados y constancias de atención que les permitían ser reconocidos por el estado como tales. De manera tal que para obtener el reconocimiento oficial era necesario que ellos objetivaran su sufrimiento mediante esas tecnologías de registro.

El Servicio de Estrés Postraumático del Hospital Alvear, único hospital de la ciudad especializado en emergencias psiquiátricas, ocupó el lugar directriz de la intervención estatal en salud mental ${ }^{5}$. El director de la sección de estrés postraumático del hospital es el Dr. Mosca, psiquiatra y presidente de la Sociedad Argentina de Psicotrauma. Entre sus antecedentes se encuentra el haber actuado en representación de los Cascos Blancos de la Cancillería Argentina -organismo estatal encargado de diseñar y ejecutar el papel argentino en la asistencia humanitaria internacional- luego del paso del Huracán Katrina por New Orleáns, en los Estados Unidos. Del total de 932 personas que recibieron atención mental durante 2005, 617 lo hicieron en ese Servicio de Estrés Postraumático. De ese total, 234 habian sido derivadas desde la línea telefónica Salud Mental Responde, es decir que el hecho de que fueran atendidas en aquel servicio fue producto de cierta decisión estatal y no de la demanda espontánea.

Según Andújar (2006) para organizar la atención inicial de las víctimas del incendio de Cromañón, el Servicio de Estrés Postraumático apeló a una clasificación que distinguía entre quienes estuvieron expuestos en forma directa al desastre y quiénes no lo estuvieron. Esa clasificación estaba orientada por el "Manual operativo sanitario para eventos con víctimas múltiples" del Servicio de Asistencias Médicas en Emergencias (SAME) de la ciudad. Tal manual diferencia entre cuatro grupos de sujetos a considerar para la atención: víctimas primarias (los sobrevivientes que estuvieron expuestos directamente al incendio); secundarias (familiares y/o allegados afectivos); terciarias (rescatistas, personal de salud, policia); y cuaternarias (integrantes de la comunidad afectada).

Los jefes de los dispositivos de salud mental de varios hospitales de la ciudad de Buenos Aires coincidieron en que las principales patologías y

\footnotetext{
${ }^{5}$ Además de ese servicio, entre los dispositivos conformados para brindar atención mental a las víctimas se destaca el del Hospital Álvarez, en donde funciona un grupo de trabajo sobre estrés postraumático conducido por el Dr. Sivak que ha atendido a más de 200 sobrevivientes. A partir del año 2014, se sumó a la atención de las víctimas el Centro Ulloa de atención a víctimas de violaciones a los Derechos Humanos que ha trabajado con alrededor de 70 sobrevivientes. Actualmente me encuentro llevando adelante una investigación que tiene como objetivo analizar las prácticas de esos profesionales que articulan psicoanálisis y psiquiatría en su trabajo con las víctimas del incendio.
} 
desórdenes presentes en la población damnificada fueron el estrés postraumático, los trastornos de ansiedad y los síndromes depresivos (ibídem). Según el Dr. Mosca, director de la sección de estrés postraumático del hospital, se trataba de cuadros que eran similares a los que padecían las víctimas de otras tragedias de naturaleza muy divergente: "los hechos que conducen al trastorno por estrés postraumático pueden ser disímiles - no es lo mismo un accidente de tránsito que el abuso sexual o un atentado terrorista-, [pero] las reacciones y los síntomas posibles pueden generalizarse"

Tal y como he señalado anteriormente, Young (1995) señala que el trastorno por estrés postraumático es un tipo de desorden mental vinculado con el tiempo ya que la memoria pasada puede hacerse presente frente a ciertos estímulos que la convoquen. Al referirse a ese trastorno el DSM IV señala que "el acontecimiento traumático puede ser reexperimentado de varias maneras. Normalmente, el individuo tiene recuerdos recurrentes e intrusos" (APA, 1994: 435). Como parte de esa sintomatología asimilable a la que se presenta en víctimas de otros tipos de desastres, entre los sobrevivientes del incendio se destacaban los flashbacks, esto es, el retorno de imágenes y vivencias del hecho que volvían a traer sufrimiento y dolor. Ello explica los síntomas evitativos de ciertos espacios y prácticas que manifestaban las víctimas. Para lidiar con esa sintomatología, uno de cada cinco pacientes del servicio estaban medicados con antidepresivos y estabilizadores de los estados anímicos.

Sin embargo, aquel diagnóstico no era uniforme para toda la población que recibía atención mental. Según el director del servicio, en el caso de los familiares de los fallecidos "más que estrés postraumático, suelen padecer depresión (...). No es lo mismo el trauma, con sus dificultades para dormir y esa sensación de peligro inminente, que el dolor desgarrador, la sensación de que eso nunca se va a pasar, de quien ha perdido a un ser querido" ${ }^{\text {? }}$. Cabe destacar entonces que, mientras que los familiares de los fallecidos llevaban la voz pública en el movimiento de denuncia exigiendo justicia, en el marco de la atención clínica eran considerados como víctimas secundarias según la clasificación señalada arriba y sus padecimientos no siempre estaban asociados al TEPT, según los especialistas. De manera tal que aún cuando familiares y sobrevivientes del incendio fueron

\footnotetext{
6 "Las pesadillas que dejó Cromañón”. Diario Clarín, 22 de junio de 2005.

7 "El estrés y la soledad, síntomas de los sobrevivientes de Cromañón". Diario Página/12, 25 de junio de 2005.
} 
reconocidos como víctimas, a pesar de este modo común de nominarlos, sus experiencias psíquicas y padecimientos fueron diferenciados por esos expertos $^{8}$.

En ese contexto, la acción de los psicólogos y psiquiatras como especialistas estatales en salud mental, se desenvolvió en un doble registro. Ellos contribuyeron fuertemente a mejorar la situación de sufrimiento de las víctimas traumatizadas. A la vez, actuaron como agentes certificantes consagrando la veracidad del padecimiento de quienes debieron acreditar objetivamente su situación para poder acceder a la compensación económica. Al mismo tiempo que la experiencia de sufrimiento de las víctimas fue legitimada por nociones como TEPT y trauma a través de la mediación de aquellos expertos, esas prácticas profesionales oficialmente reconocidas se constituyeron en un recurso necesario para que éstas hicieran valer sus derechos frente al Estado.

\section{LA ESPERA COMO DENUNCIA: TRAUMA E IMPUNIDAD}

Muchas de las víctimas que recibieron atención psicológica formaban parte del movimiento Cromañón, el colectivo que entre 2004 y 2009 llevó adelante una lucha política y pública en las calles de Buenos Aires reclamando que se encarcelara a los responsables del incendio. Además de las víctimas participan allí otros actores que las acompañan. Se trata de profesionales psicólogos, psicólogos sociales, psicoanalistas y psiquiatras. Dadas las consecuencias traumáticas y dolorosas que ha dejado el incendio en familiares y sobrevivientes, su presencia siempre fue considerada como una cuestión normal ya que brindan contención afectiva, actúan como intermediarios cuando hay conflictos, aportan recursos y conocimientos, etc. Su presencia es valorada especialmente en las fechas clave como los aniversarios y cumpleaños que pueden funcionar como estímulos para que se desencadene un proceso traumático. Por eso es habitual encontrarlos entre las víctimas en esas circunstancias especiales. Como señalé al principio, ellos fueron quienes denunciaron que la espera por la realización del juicio penal que estaban atravesando las víctimas, era una expresión de impunidad y las sumía en el dolor. La relación trazada allí entre espera y

\footnotetext{
${ }^{8}$ Para un examen detallado de los contrastantes circuitos burocráticos de acreditación de la condición de víctima seguidos por sobrevivientes y por familiares de los fallecidos, véase mi etnografía, Zenobi (2014). Sobre el papel de los expertos en catástrofes y de los sociólogos estadísticos en ese proceso, véase Zenobi (2017).
} 
sufrimiento me obliga a indagar en el aspecto temporal de este proceso 9 . Me inspiro en las palabras de Gell (1992) para quien una antropología del tiempo debe dar cuenta de cuándo, cómo y porqué el tiempo deviene una cuestión relevante en ciertos asuntos prácticos con los que lidian los seres humanos.

La realización del juicio penal en el que se determinarian quienes fueron los responsables de las 194 muertes, estaba propuesta para inicios de 2008. Sin embargo, en el mes de abril de ese año desde el poder judicial se aseguró que no estaban dadas las condiciones materiales para alojar a las cientos de víctimas que tenían derecho a presenciar las audiencias públicas. La demora en el inicio del juicio preocupaba a los familiares de los fallecidos que exigían justicia, por lo que realizaron un evento para presionar al Estado. Así fue que impulsaron la realización de una conferencia de prensa que tuvo como consigna "La falta de Justicia y la impunidad, nos están matando". Al realizar declaraciones en diversos medios de comunicación para difundir la conferencia, los familiares declararon a la prensa que varios años después del incendio, el TEPT estaba conduciendo a la muerte a numerosas personas vinculadas al hecho: "El estrés postraumático ha empezado a cobrarse vidas: a la extensa lista de 194 víctimas del 30 de diciembre de 2004 se han agregado 2 sobrevivientes, 6 madres, 2 padres, más de 30 abuelos en los 39 meses ya transcurridos". Según esa consigna de denuncia política, 39 meses después del incendio 79 personas más habian fallecido como consecuencia del TEPT.

La conferencia de prensa se realizó en un hotel céntrico de la ciudad de Buenos Aires. Se trataba de un pequeño salón en el que había reunidos unos treinta familiares de los muertos y unos pocos sobrevivientes. El pequeño recinto estaba desbordado por cámaras de televisión y una decena de periodistas de radio, TV y de la prensa gráfica. De frente al público se encontraba una mesa con seis sillas. Como apertura, uno de los líderes del movimiento introdujo el motivo que los había convocado a dar la conferencia:

(...) necesitamos que se haga el juicio porque estamos padeciendo las consecuencias de que no haya justicia (...). Esto tiene un apoyo científico. Para eso en esta conferencia de prensa nos acompañan profesionales: la Dra. Ana Quiroga, Directora de la Escuela de Psicología Social Pichón Riviere; la Dra. María

\footnotetext{
${ }^{9}$ Para algunas interesantes etnografías sobre situaciones de espera, ver Comfort (2008) y Kobelinsky (2010).
} 
Casariego, Miembro de la Asociación Argentina de Psicología (APA) y Miembro de la Escuela de Psicoterapia para Graduados; el Dr. Alfredo Grande, psiquiatra y miembro de la Liga Argentina por los derechos del Hombre; y la Dra. Diana Kordon, directora del Equipo Argentino de Investigaciones Psicosociales. Ellos pueden dar razón de la relación entre muerte y angustia por no tener justicia. (Conferencia de prensa de familiares $y$ sobrevivientes de Cromañón, Buenos Aires, 14 de abril de 2008).

La intención de los familiares al invitar a estos profesionales psi, era fuertemente estratégica: ellos creían que desde algunos sectores de la sociedad argentina sus voces sufrientes y traumatizadas eran percibidas como irracionales (Zenobi, 2013). La intervención de esos especialistas no sólo venía a legitimar su condición de víctimas sino que a través de la participación de esos profesionales comprometidos con su causa, se proponían interpelar al Estado y exigir su derecho a que se realizara el juicio penal sobre la base de fundamentos científicos.

Al observar el perfil de los profesionales psi invitados a dar la conferencia, puede verse que la elección no fue casual, sino que se trató de profesionales reconocidos en el campo de la militancia por los derechos humanos en Argentina. En ese país, la relación entre militancia política y profesionales comprometidos con la causa de los derechos humanos cuenta con una larga historia. Ellos articulan el cruce entre política y técnica y así construyen sus posiciones tanto en el campo de la militancia como en el campo propio de su saber específico ${ }^{10}$. En el caso de la psicología, el cruce con la cultura de izquierda iniciado durante la década de 1960 y la militancia política activa durante los años 1970 - que implicó la dolorosa desaparición de más de 200 trabajadores de la salud mental durante la dictadura militar-, sentó las bases de ese compromiso político público (Noailles, 2010). Algunos de estos profesionales comprometidos eran los protagonistas del acto organizado por los familiares de los fallecidos.

La primera intervención en la conferencia de prensa fue la de una reconocida profesional del campo psi con una amplia trayectoria en el campo de los derechos humanos. Diana Kordon es médica, psiquiatra y psicoterapeuta. Durante la década de 1980 coordinó el equipo de asistencia psicológica de la Asociación Madres de Plaza de Mayo. Es

\footnotetext{
${ }^{10}$ Para el caso de los profesionales del derecho comprometidos con la causa de los Derechos Humanos, ver Vecchioli (2006).
} 
docente de grado y posgrado en diferentes instituciones nacionales, coautora de varios libros sobre salud mental y derechos humanos como "Efectos psicológicos y psicosociales de la represión política y la impunidad" (Kordon, 2005), y fue ganadora de una beca Guggenheim vinculada a ese tema ${ }^{11}$. Actualmente es coordinadora del Equipo Argentino de Trabajo e Investigación Psicosocial, una institución de salud mental y derechos humanos. Al hablar en la conferencia de prensa, Kordon señaló que:

(...) nada va a tirar atrás lo ocurrido, nada va a devolver a un hijo, nada va a devolver a los sobrevivientes el terror de lo vivido, pero la justicia sí puede cumplir un papel de reparación simbólica. Puede definir que hubo un daño que se produjo, puede decir que hubo responsables por ese daño, que eso que ocurrió debe ser sancionado, puede decir qué es lo permitido y qué es lo prohibido. Todo esto es lo que no ocurre con la impunidad, por eso la impunidad es una nueva retraumatización para los familiares, la falta de justicia implica un sufrimiento (...) el Estado tiene la obligación de responder porque eso implica abrir verdaderamente el camino de la elaboración personal y social de lo ocurrido. (Conferencia de prensa de familiares y sobrevivientes de Cromañón, Buenos Aires, 14 de abril de 2008).

Así, Kordon habló del papel "reparador"12 de la justicia, destacando la necesidad de evitar una situación de impunidad que condujera a que las víctimas fueran retraumatizadas. El trauma y el dolor asociado a ello, estaban latente y podía regresar. Como señala esta profesional en su libro en coautoría dedicado a su trabajo con víctimas de la dictadura militar, titulado "La impunidad: Reactivación del trauma psíquico":

\footnotetext{
${ }^{11}$ Sobre la relación entre saberes psi y víctimas de dictaduras militares como sujetos traumatizados, para el caso de Brasil, ver Aydos y Figueredo (2013).

12 El concepto de "reparación simbólica" es parte central del lenguaje y de las herramientas jurídicas que se movilizan en el campo de la lucha por los derechos humanos en todo el mundo. En Latinoamérica, desde la década de 1980 el concepto ha sido fuertemente impulsado por la Comisión interamericana de Derechos humanos, que por su carácter transnacional contribuye a modular los lenguajes locales de las intervenciones jurídicas. En relación a esto, cabe destacar el caso del Centro de Estudios Legales y Sociales (CELS) - uno de los organismos de derechos humanos más importantes de Argentina y de la región- que cuenta con un equipo de salud mental que, en su documento guía sobre reparación simbólica sostiene que: "el reconocimiento público y el accionar de la justicia, es decir, el pasaje de lo privado e íntimo como sufrimiento a la esfera de lo público, restituyen algo de la trama que fue lesionada por el trauma (...) No puede negarse (...) el efecto de apaciguamiento del dolor que un acto de justicia produce sobre quien ha sufrido una situación traumática, sobre todo si ésta ha sido producida por el Estado" (Guilis y Equipo de salud mental del CELS, s/f: 10).
} 
"Al no haber justicia el proceso de elaboración de la situación traumática se realiza en condiciones sociales que, lejos de suprimir los factores traumáticos, los refuerza por la situación de impunidad (...). En nuestra práctica asistencial hemos observado cómo con cada nueva medida política o jurídica relacionada con la impunidad recrudecen en muchos de nuestros asistidos la angustia o la sintomatología" (Edelman, Kordon y Lagos, 1995: 25).

Entre los profesionales comprometidos con los derechos humanos que participaron de la conferencia de prensa también se encontraba Alfredo Grande, un reconocido psiquiatra y psicoanalista, integrante del directorio de uno de los organismos más antiguos de derechos humanos de la Argentina: la Liga argentina por los Derechos del Hombre. Docente universitario, promueve un psicoanálisis implicado con lo social y en los cursos que brinda en universidades argentinas articula práctica profesional y política. En su intervención durante la conferencia de prensa destacó que “hay impunidad cultural cuando pasó algo terrible pero para la sociedad es como si no hubiera pasado nada (...). La impunidad mata, la impunidad cultural mata y ese es el momento que estamos atravesando". Como Kordon, este profesional militante también contribuyó a trazar una relación entre sufrimiento e impunidad. Al referirse al "momento que estamos atravesando" hablaba de la espera a la que estaban siendo sometidas las víctimas del incendio de Cromañón por el Estado que no avanzaba hacia la administración de justicia. Según sus palabras, esto conducía al dolor y a la muerte de quienes estaban esperando la pronta realización del juicio penal.

Como parte del lenguaje propio de la denuncia política, durante el evento que describo, tanto las víctimas como los expertos que dan vida a esta configuración social movilizaron las nociones de trauma y estrés postraumático — más allá de las diferencias y matices que cada una implica desde el punto de vista técnico-, y otras como elaboración y reparación simbólica. Unos y otros señalaron que cuatro años después del incendio, las víctimas continuaban esperando justicia. Su situación era representada como ubicada en un tiempo intermedio entre dos momentos -en el presente, entre el pasado y el futuro-: el incendio de Cromañón como el evento traumático fundante y la sentencia de la causa penal. El proceso psíquico y emocional que estaban atravesando estaba abierto, desenvolviéndose en el tiempo: la herida abierta (en el pasado) luego del suceso traumático, aún no había cicatrizado y nadie sabía si eso ocurriría 
(en el futuro) ni cómo. La institución judicial tenía la posibilidad de contribuir a esa cicatrización al dar reparación simbólica a los sufrientes. En cambio, si la resolución era negativa a los intereses de las víctimas, ellas se verían traumatizadas nuevamente. La conexión entre trauma e impunidad implicaba que el sufrimiento traumático podía reaparecer bajo ciertas condiciones o estímulos que podían darse a lo largo del tiempo. Si esa relación entre trauma e impunidad podía ser construida en la denuncia pública ello se debía al aspecto temporal inscripto en la noción de trauma psíquico, aspecto destacado por Young (1995) y al que me he referido más arriba.

Al abordar las relaciones entre Estado y temporalidad varios estudios sociológicos han destacado el vínculo existente entre espera y poder. Inspirado en Bourdieu (1999) y en Schwartz (1974) para quienes esperar es uno de los modos más característicos de experimentar el poder a través de la dependencia y la subordinación, Auyero señala que los peregrinajes de las personas por las burocracias estatales son "procesos temporales a través de los cuales la subordinación política es reproducida" (2012: 2). A través de estas lecciones cotidianas "la espera objetiva se convierte en sumisión subjetiva" (ibídem: 28) y es así como las personas se convertirian en "pacientes del Estado". En cambio, en su excelente trabajo sobre India, Mathur señala que "en la espera las personas se convierten en los más fuertes y articulados críticos del Estado" (2014: 151). Desde esta mirada, la espera no es solo algo que se sufre sino que también se comprende, se explica y se denuncia. Entonces vemos a las personas debatiendo e impugnando al Estado, justamente, porque no están dispuestos a continuar esperando. La im-paciencia se impone sobre el hecho de ser un "paciente del Estado". Aqui he mostrado que como parte de su activismo denunciante de la espera, las víctimas del incendio de Cromañón convocaron a ciertos profesionales psi con trayectorias en el campo de los derechos humanos que fundamentaron a través de sus credenciales profesionales pero también de su experiencia y militancia por los derechos humanos, sus exigencias y demandas. Se trataba de legitimar su situación para demandar que se realizara el juicio. Esta situación se repite en otros casos y contextos en los que de igual manera, "gracias a la mediación de los expertos, las víctimas, cuyo status está autentificado por el traumatismo, utilizan a este como un recurso político para hacer valer sus derechos" (Latté y Rechtman, 2006: 167). 
Mientras que desde la atención clínica los especialistas de la salud mental contribuyeron a validar la condición de víctimas frente a las instancias burocráticas del Estado, ahora, a través de sus intervenciones políticas en el espacio público, algunos de estos profesionales contribuían a establecer una relación entre una categoría diagnostica como trauma y una categoría moral: las víctimas de Cromañón.

\section{LA ESPERA COMO DEFENSA: EL PROCESO PSÍQUICO Y EL PROCESO JUDICIAL}

Durante la espera por la realización del juicio penal, las víctimas del movimiento Cromañón tuvieron numerosas tensiones con el gobierno de la ciudad que estuvieron relacionadas principalmente con el santuario. El santuario era el espacio conmemorativo edificado espontáneamente sobre la calle donde se depositaron los cientos de cadáveres la noche del incendio. Ese memorial era un espacio de ritulización y performance para la protesta de los demandantes, del mismo modo que ocurre en otros contextos (Margry y Sánchez-Carretero, 2011). El mismo representaba un problema para el Estado local porque al estar construido en medio de una calle, interrumpía el tránsito vehicular y generaba un caos en el centro de la ciudad. Mientras que el gobierno regularmente amenazaba con retirarlo, las víctimas sostenían que el memorial debía quedar en su lugar.

Las categorías e interpretaciones emocionales y psicológicas para oponerse a que se retirara el santuario circularon fuertemente entre las propias víctimas. Una de las explicaciones centrales que circulaban entre ellas refería a la necesidad de realizar adecuadamente el "proceso psicológico". Los familiares de los fallecidos decían que antes de retirar el santuario y reabrir la calle "primero hay que hacer un trabajo psicológico, porque aún no sanó el alma de ninguno de los relacionados con la masacre"13. El santuario debía quedar todavía en su lugar porque para abrir la calle al tránsito vehicular "hay que tener un tiempo de maduración. Para hacer el museo del Holocausto se tardó 50 años (...) creemos que debe haber una maduración para retirar el santuario"14. De modo tal que si antes se había movilizado la idea de "espera" para denunciar la demora en la realización del juicio, la defensa del memorial implicaba sostener que era necesario mantener la "espera" hasta que se definiera la situación judicial.

\footnotetext{
13 "Cromagnon: estudian reabrir una calle". Diario La Nación, 26 de mayo de 2007.

14 "Un proyecto para Cromañón". Diario Página/12, 28 de mayo de 2007.
} 
Debido a las frecuentes tensiones con el gobierno, una semana después de la conferencia de prensa denunciando la espera y pidiendo que se realizara el juicio penal, el movimiento Cromañón convocó a nueva conferencia de prensa para explicitar de modo firme su posición sobre el santuario. Frente a los numerosos medios de prensa allí reunidos, los referentes del colectivo señalaron que retirarian el memorial "cuando se llegue a la sentencia de condena y se haga justicia, ese será el momento. Pedimos que nos dejen hacer nuestro duelo, y que nos dejen decidir cómo hacer memoria". Lo que estaban diciendo allí era que el santuario vería su fin cuando terminase la espera: cuando la justicia hiciera justicia, esto es, cuando el juicio penal tuviera una resolución favorable a sus intereses. Esta era la condición necesaria para que el proceso psíquico avance positivamente y las víctimas estén en condiciones de desarmar el memorial.

Términos y expresiones como "tiempo de maduración", "trabajo sicológico", "proceso psíquico", remiten inevitablemente al carácter temporal del sufrimiento. Para los actores sociales que dan vida a esta figuración social, la temporalidad del proceso psíquico no es estrictamente "interna" sino que está intimamente relacionada con la lucha por conseguir justicia. Como afirma Gell "las representaciones colectivas sobre el tiempo sólo cobran coherencia a la luz de sus relaciones implícitas con lo práctico" (1992: 326). De modo tal que desde esa mirada, habría una coordinación entre procesos de diferente orden: el proceso psíquico y el proceso judicial. Un ejemplo claro de esta perspectiva de las víctimas es el documento público elaborado por el movimiento unos años antes que llevaba como título "La lucha no es locura, la impunidad enloquece", y en donde se señalaba: "el día que (...) los responsables vayan presos y paguen por esta masacre, entonces sí, Señora Jueza, podremos tener un duelo normal"15.

El campo de la salud mental no fue ajeno a las disputas por el santuario que se dieron entre el movimiento y el Estado durante la espera por el juicio penal. El tema llegó a tratarse en el Congreso Internacional de Trauma Psíquico y Estrés Traumático. Se trata de un evento organizado por la Sociedad Argentina de Psicotrauma, la Sociedad Internacional de Estudios sobre el Estrés Traumático y la Asociación Mundial de Psiquiatría, que tuvo ediciones en ciudades como Amsterdam, Jerusalén y Melbourne. En esos encuentros se debate acerca de la asistencia a víctimas de abuso, accidentes, etc., pero también de situaciones como el huracán Katrina, el

${ }^{15}$ Documento difundido por el movimiento Cromañón en la conmemoración de los 22 meses del incendio, octubre 2006. 
11-S y la invasión a Irak, el Holocausto, el Tsunami del sudeste asiático y la Guerra de Malvinas. En la edición del año 2008 realizada en Buenos Aires, el presidente del congreso fue el Dr. Daniel Mosca, director del Servicio de Estrés Postraumático del hospital de urgencias psiquiátricas, que como he señalado antes fue uno de los principales dispositivos de atención en salud mental a las víctimas del incendio. En el congreso hubo una mesa redonda dedicada especialmente a Cromañón, que llevó como título: "Tragedia de Cromañón. Consecuencias Físicas de las Consecuencias Psíquicas".

Los especialistas que hablaron en esa mesa de trabajo trazaron una relación entre trauma e impunidad. El primero de ellos fue alguien que ya había participado en la conferencia de prensa en la que las víctimas solicitaban la pronta realización del proceso penal. Se trató del Dr. Alfredo Grande quien afirmó que "las consecuencias físicas de estos traumas no elaborados son distintas enfermedades, incluso cáncer" ${ }^{16}$. El segundo orador fue el Dr. Fariña, Investigador Principal de la Universidad de Buenos Aires y Profesor Titular de la asignatura Psicología, Ética y Derechos Humanos de la carrera de psicología, quien señaló la necesidad de que se respete el proceso de duelo por el que se encontraban atravesando las víctimas: "el tratamiento que se haga del espacio público no es indiferente al cuerpo sufriente de cada uno de aquellos que están llorando esas pérdidas (...) En Cromañón la calle permanece cerrada en señal de duelo". Así los expertos en salud mental hicieron pública su opinión autorizada sobre la necesidad de mantener el santuario en su lugar: retirar compulsivamente ese espacio conmemorativo podía traer consecuencias psíquicas y físicas negativas para los familiares de los muertos y sobrevivientes del hecho. Si antes había sido necesario denunciar la espera por el juicio, ahora que el juicio había comenzado era necesario esperar la resolución judicial para resolver qué hacer con el santuario.

En su trabajo sobre la antropología del tiempo, Gell (1992) debate tanto con las formulaciones durkheimnianas acerca de la temporalidad según las cuales los modos de concebir el tiempo son esencialmente sociales, como con los enfoques inspirados en la filosofía de la experiencia que enfatizan en las experiencias subjetivas. Él, en cambio, insiste en distinguir entre el tiempo y los procesos que ocurren en el tiempo: lo central de una categoría abstracta como "tiempo" es que provee los medios para la unificación de

16 "Cromañón: Consecuencias físicas de las consecuencias psíquicas". Agencia de noticias Indimedia, 27 de junio de 2008 . Disponible en: http://argentina.indymedia.org/news/2008/06/611192.php. Última consulta: 10/06/2016. 
diferentes categorías de procesos: "El tiempo -que es intrínsecamente unitario y unificado-, permite la coordinación de diversos procesos; biológicos con sociales, psicológicos o subjetivos con objetivos" (ibídem: 316).

Puede verse en el caso analizado que durante el tiempo que se desenvolvió la espera, se dio una coordinación entre el proceso psicológico y el proceso penal. Tanto desde el punto de vista de las víctimas, como desde el de los especialistas de la salud mental existía una coordinación entre el sufrimiento, el trauma, el estrés postraumático - todos modos de nombrar la experiencia de las víctimas- y los caminos que seguía la administración estatal de justicia. Si se hacía justicia habría alivio y reparación, pero si se iba en el camino contrario, esos padecimientos volverían y se verían amplificados por la situación de impunidad. Este modo de representarse la espera a través de la conexión entre dos procesos de naturaleza diferente indica que, como sostiene Gell (ibídem), las nociones sobre el tiempo sólo pueden entenderse a la luz de los asuntos prácticos con los que lidian los seres humanos.

Ahora bien, ¿cómo se terminó definiendo la espera de las víctimas? Entre agosto de 2008 y agosto de 2009 se realizó el juicio penal para deslindar responsabilidades por el incendio. La espera estaba llegando a su fin. El día en que se leyó la sentencia final, en la sala había cientos de sobrevivientes y de familiares de los fallecidos. Concentrados en la puerta del tribual ubicado en el centro de Buenos Aires había alrededor de mil personas expectantes por conocer la definición de la situación. Entre ellos había algunos profesionales de la salud mental que acompañan a las víctimas, ya que el día de la sentencia era visto por todos como un posible estímulo que podía funcionar como un desencadenante para la reactualización del trauma y el sufrimiento.

Durante la lectura de la sentencia los jueces otorgaron penas de prisión muy bajas a quienes las víctimas veían como responsables del incendio. Además, muchas de esas penas contemplaban la posibilidad de la excarcelación. Compungidos por la situación de impunidad que acababa de definirse judicialmente, instantes después de la lectura de la sentencia comenzaron a desatarse incidentes y disturbios entre la policía y quienes estaban reunidos en la calle. La situación era caótica, entre llantos y desesperación había personas corriendo y arrojando objetos contra los efectivos policiales que reprimían sin ninguna consideración hacia las víctimas, con bastones y camiones hidrantes. 
Terminada la espera, semanas después de finalizado el proceso judicial, nuevamente renacieron los conflictos con el gobierno de la ciudad cuando sus funcionarios manifestaron su intención de retirar el santuario. Las víctimas informaron su rechazo a esta iniciativa e insistieron en que el espacio de conmemoración debía permanecer en su sitio. Debido a que la resolución del juicio había sido contraria a sus expectativas, no se había hecho justicia y había ganado la impunidad. Por ello, la situación psíquica y anímica de las víctimas era considerada peor que antes, por lo que éstas no estaban en condiciones emocionales de permitir que se retirase aquel memorial. En una carta que dirigieron a los funcionarios del gobierno denunciaron que:

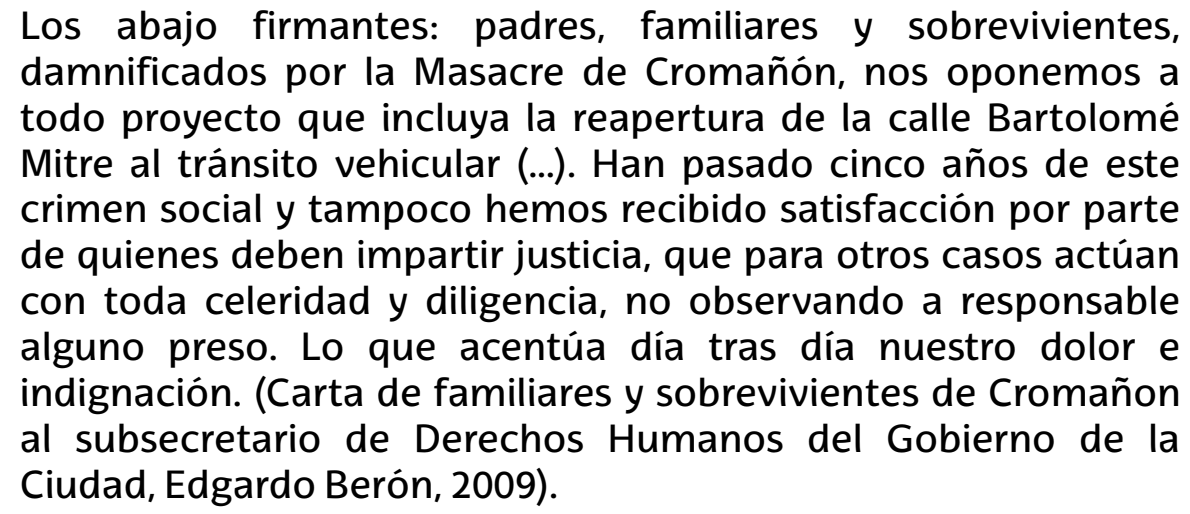

La conexión entre el proceso psíquico y el proceso penal que se desenvolvió durante el tiempo de la espera, justificó en parte, junto a otros argumentos de orden político, que el santuario no fuera retirado de su lugar. Ahora había que continuar esperando a que las instancias judiciales superiores a las que apelarian las víctimas, resolvieran definitivamente la situación de los acusados ${ }^{17}$.

\section{CONCLUSIONES: LAS CATEGORÍAS PSI, DESDE Y HACIA EL ESTADO}

La centralidad de la condición de víctima en las sociedades actuales está relacionada con la valoración moral y la eficacia de esa figura al momento de exigir reconocimiento social y reparación por el sufrimiento vivido. Frecuentemente las comunidades y colectivos de personas que han vivido las desgracias más diversas, ponen en juego categorías de orden psi como "trauma" que vienen a autenticar la gravedad de lo sufrido. Los diferentes

\footnotetext{
${ }^{17}$ Para un análisis in extenso del recorrido del espacio conmemorativo construido por las víctimas del incendio desde su formación y posterior modificación, hasta su situación actual, ver Zenobi (2016).
} 
tipos de víctimas alrededor del globo que apelan a categorías psi para explicar(se) su situación se esfuerzan porque el sufrimiento sea comprendido por el resto de los miembros de la comunidad a través de un lenguaje que se supone común (Mauss, 2005). Este fenómeno no hubiese sido posible sin la reconversión que el concepto de trauma ha tenido en la segunda mitad del siglo pasado cuando "el traumatismo pasó de ser un signo de infamia a una fuente de reconocimiento. Esta impresionante reconversión procede menos de un descubrimiento médico que de una movilización política" (Fassin, 2014: 170).

En su propuesta acerca de los dispositivos que contribuyen a la producción social de las víctimas, Dodier (2015) señala que debemos prestar atención al papel que cumplen en ese proceso y a cómo se relacionan entre sí diversos dispositivos. En este caso he mostrado cómo luego del incendio las políticas públicas de atención, el subsidio económico, el memorial, el juicio penal, fueron dispositivos en torno de los cuales las víctimas y los especialistas de la salud mental tramaron relaciones de interdependencia y dieron vida a una cierta figuración social. En ese escenario, abordé el papel de los profesionales psi como agentes activos que contribuyeron a modelar la mirada de las propias víctimas sobre sí mismas y la mirada pública sobre el incendio como un hecho traumático.

En primer lugar, eso fue posible a través de sus intervenciones clínicas en el marco de la atención estatal a las víctimas. Al privilegiar un Servicio de Estrés Postraumático como institución directriz de sus intervenciones, el Estado local fue un importantísimo actor en la clasificación de la situación traumática. Pero, y en segundo término, los saberes psi también fueron movilizados en un sentido opuesto: ya no desde el Estado con un sentido terapéutico sino, al revés, desde el movimiento Cromañón para denunciar al Estado. Las intervenciones de los profesionales psi que expusieron su compromiso con la causa de los sufrientes implicaban un cruce entre la posesión de credenciales profesionales y los imperativos morales y políticos propios de la lucha en demanda de justicia. Las víctimas encontraron en saberes como la psiquiatría o la psicología, al mismo tiempo, por un lado, un recurso para ser asistidos y contenidos por profesionales $y$, por el otro, herramientas para legitimar su causa y sus demandas públicas. Toda esta movilización de los saberes psi estuvo enmarcada por la consideración extendida en ciertos sectores urbanos argentinos sobre ese lenguaje como una explicación razonable del sufrimiento. 
Si bien se ha enfatizado en el aspecto individualizante, privatizador de la experiencia de sufrimiento que suelen implicar las clasificaciones clínicas, pueden verse aquí los usos socialmente creativos a través de los cuales se da la construcción de comunidades que se organizan para impugnar y denunciar. Categorías diagnósticas que forman parte de un cierto universo profesional fueron utilizadas para la movilización política más allá de los sentidos que se le puede dar en la descripción clínica: estos usos políticos del trauma implican "la importación de una categoría psiquiátrica al tratamiento político de un desastre colectivo. Trauma ya no es simplemente una realidad clínica individual, sino que deviene un vector de identificación colectiva" (Latté y Rechtman, 2006: 175). Al destacar este proceso de "importación" cabe resaltar algunos contrastes entre los usos expertos de las categorías clínicas y sus usos sociales. Al respecto vemos que mientras que los familiares de los fallecidos fueron considerados como víctimas "secundarias" según el protocolo de atención en emergencias, y que según los especialistas sus padecimientos no solían estar relacionados con el Trastorno por Estrés Postraumático, en cambio, fueron ellos y no los jóvenes sobrevivientes quienes llevaron la voz pública protagónica en el movimiento de denuncia exigiendo justicia.

Apoyándose en la misma matriz interpretativa que los expertos - matriz que también forma parte de la cotidianeidad en amplios sectores de la sociedad argentina-, términos tales como trauma, duelo patológico, proceso psíquico y estrés postraumático, estuvieron de la mano con dolor, indignación y sufrimiento alimentando el lenguaje de la lucha. Siguiendo el camino al que nos conduce el uso de esas categorias, vemos que a lo largo del tiempo de la espera, se articularon dos procesos de naturaleza diferente: el proceso psíquico y el proceso penal. La experiencia de padecimiento de las víctimas no era estrictamente de carácter individual, sino que estaba ligada a su condición de actores movilizados en una causa pública. Por esto, la superación del padecimiento no dependía sólo de ellos ni tampoco sólo de los profesionales psi del Servicio de estrés postraumático que les dieron asistencia. En cambio, dependía también de las decisiones de otros actores como abogados, fiscales y jueces que debían brindar reparación simbólica. Tanto la denuncia de la "espera" por el juicio, así como la defensa de la "espera" para oponerse a que el Estado retirara el santuario, refieren a la coordinación entre aquellos procesos. En ambos casos, lejos de quedar ubicadas en una situación pasiva, durante el 
tiempo de espera las víctimas activaron formas de lucha y resistencia novedosas.

Si recuperamos las relaciones de diferente tipo que las víctimas del incendio establecieron con el Estado, debemos tener en cuenta que, por un lado, ellas lo denunciaron al realizar la conferencia de prensa cuando se sintieron amenazadas a causa de la dilatada espera por la realización del juicio. A su vez, paradójicamente, esas personas habían sido consagradas socialmente como víctimas traumatizadas por ese mismo Estado a través de la intervención de sus profesionales que les dieron atención clínica y que actuaron certificando sus padecimientos para que pudieran acceder a cobrar el subsidio económico. Contra algunas miradas romantizadas centradas en las luchas y las resistencias que plantean las víctimas en el mundo contemporáneo, este tipo de procesos cruzados nos muestran que a nivel analítico debemos evitar tomar como puntos de partida dicotomías como aquella que opone Estado y sociedad civil. En ese camino, una orientación eliasiana puede contribuir a enfocar la mirada hacia aquellos procesos que hablan de las relaciones de interdependencia entre las personas concretas que dan vida a las figuraciones sociales particulares como la que aquí me ha ocupado.

\section{BibLIOGRAFÍA}

American Psychiatric Association (1980). Diagnostic and Statistical Manual of Mental Disorders III. Washington: American Psychiatric Association publication office.

American Psychiatric Association (1994). Diagnostic and Statistical Manual of Mental Disorders IV. Washington: American Psychiatric Association publication office.

Andújar, A. (2006). República Cromagnón. A dos años de la tragedia: una evaluación de las políticas públicas del Gobierno de la Ciudad Autónoma de Buenos Aires. Buenos Aires: Legislatura de la Ciudad de Buenos Aires.

Auyero, J. (2012). Patients of the State: The Politics of Waiting in Argentina. Durham: Duke University Press. 
Aydos, V., \& Figueiredo, A. (2013). A construção social das vítimas da ditadura militar e a sua ressignificação política. Interseções: Revista de Estudos Interdisciplinares, 15(2), 392-416.

Bear, L. (2014). Doubt, Conflict, Mediation: The Anthropology of Modern Time. Journal of the Royal Anthropological Institute, special issue, 3(30), 1-28.

Bourdieu, P. (1999). Meditaciones pascalianas. Barcelona: Anagrama.

Caple James, E. (2010). Democratic Insecurities: Violence, Trauma, and Intervention in Haiti. California: University of California Press.

Comfort, M. (2008). Doing Time Together: Love and Family in the Shadow of the Prison. Chicago: University of Chicago Press.

d'Halluin, E. et al. (2004). La deuxième vie du traumatisme psychique. Cellules médico-psychologiques et interventions psychiatriques humanitaires. Revue française des affaires sociales, 1, 57 -75.

Dodier, N. (2015). Postscript: thinking (by way of) disaster. In S. Revet \& J. Langumier (Eds.), Governing Disasters Beyond Risk Culture (pp. 221244). Nueva York: Palgrave McMillan.

Edelman, L., Kordon, D., \& Lagos, D. (1995). La impunidad: reactivación del trauma psíquico. Reflexión (CINTRAS-Centro de salud mental y derechos humanos), 24, 24-26.

Elias, N. (1980). La soledad de los moribundos. México: Fondo de cultura económica.

Elias, N. (1982). La sociedad cortesana. México: Fondo de cultura económica.

Fassin, D. (2014). De l'invention du traumatisme à la reconnaissance des victimes. Genèse et transformations d'une condition morale. Vingtième siècle. Revue d'histoire, 123, 161-171.

Fassin, D., \& Rechtman, R. (Eds.) (2007). L'Empire du traumatisme. Enquête sur la condition de victime. Paris: Flammarion.

Gell, A. (1992). The Anthropology of Time: Cultural Constructions of Temporal Maps and Images. Oxford: Berg.

Guilis, G., \& Equipo de salud mental del CELS (s/f). El concepto de reparación simbólica. Disponible en: http://www.cels.org.ar/common/documentos/concepto_reparacion simbolica.doc.

Kobelinsky, C. (2010). L'accueil des demandeurs d'asile. Une ethnographie de l'attente. París: Du Cygne. 
Kordon, D. et al. (2005). Efectos psicológicos y psicosociales de la represión politica y la impunidad. Buenos Aires: Asociación Madres de Plaza de Mayo.

Latté, S., \& Rechtman, R. (2006). Enquête sur les usages sociaux du traumatisme à la suite de l'accident de l'usine AZF à Toulouse. Politix, 1(73), 159-184.

Mathur, N. (2014). The reign of terror of the big cat: bureaucracy and the mediation of social times in the Indian Himalaya. Journal of the Royal Anthropological Institute, 3(30), special issue coordinated by L. Bear, 148-165.

Margry, P., \& Sánchez-Carretero, C. (2011). Grassroots Memorials. The Politics of Memorializing Traumatic Death. Nueva York: Berghahn books.

Mauss, M. (2005). A expressão obrigatoria dos sentimentos. In Ensaios de sociología (pp. 315-335). San Paulo: Editora perspectiva.

Noailles, G. (2010). Estudiantes, militantes, analistas. Historia y política de la Carrera de psicología en la Universidad de Buenos Aires. Buenos Aires: Koyatún Editorial.

Plotkin, M. (2001). Freud in the Pampas. The Emergence and Development of a Psychoanalytic Culture in Argentina. Stanford: Stanford University Press.

Plotkin, S., \& Visacovsky, S. (2007). Saber y autoridad: intervenciones de psicoanalistas en torno a la crisis en la Argentina. EIAL: Estudios Interdisciplinarios de America Latina y el Caribe, 18(1), 13-40.

Robben, A. (2005). Political Violence and Trauma in Argentina. Filadelfia: University of Pennsylvania Press.

Russo, J., \& Venâncio, A. (2006). Classificando as pessoas e suas perturbações: a 'revolução terminológica' do DSM III. Revista Latinoamericana de Psicopatologia Fundamental, IX(3), 460-483.

Sarti, C. (2011). A vítima como figura contemporânea. Caderno CRH, 24(61), 51-61

Schwartz, B. (1974). Waiting, Exchange, and Power: The Distribution of Time in Social Systems. American Journal of Sociology, 79(4), 841-870.

Seagal, L. B. (2014). Why is Muna crying?: event, relation, and immediacy as criteria for acknowledging suffering in Palestine. In H. Rønsbo \& S. Jensen (Eds.) Histories of Victimhood (pp. 179-197). Filadelfia: University of Pennsylvania Press. 
Vecchioli, V. (2006). A luta pelo direito: engajamento militante e profissionalização dos advogados na causa pelos direitos humanos na Argentina. Tese de Doutorado em Antropologia. Río de Janeiro: Universidade Federal do Rio de Janeiro.

Visacovsky, S. (2002). El Lanús. Memoria y política en la construcción de una tradición psiquiátrica y psicoanalítica argentina. Madrid/Buenos Aires: Alianza Editorial.

Visacovsky, S. (2009). La constitución de un sentido práctico del malestar cotidiano y el lugar del psicoanálisis en la Argentina. Cuicuilco, 16(45), 51-78.

Young, A. (1995). The harmony of illusions: inventing post-traumatic stress disorder. Princeton: Princeton University Press.

Zenobi, D. (2013). Del "dolor" a los "desbordes violentos". Un análisis etnográfico de las emociones en el movimiento Cromañón. Intersecciones en antropología, 14(2), 353-367.

Zenobi, D. (2014). Familia, política y emociones. Las víctimas de Cromañón entre el movimiento y el Estado. Buenos Aires: Antropofagia.

Zenobi, D. (2016). Victimes, politiciens et experts face au massacre du Cromañón. In D. Zenobi, S. Ullberg \& T. Camargo da Silva (Eds.). Traces. Memoires des crises en Amerique Latine (pp. 103-124). Paris: L'Harmattan.

Zenobi, D. (2017). Políticas Para La Tragedia: Estado y expertos en situaciones de crisis. Iberoamericana - Nordic Journal of Latin American and Caribbean Studies (Special Issue: La contribución de la Antropología al estudio de crisis y desastres en América Latina. S. Ullberg Ed.), 46(1), 30-41. 